\title{
Engineering space for light via transformation optics
}

\author{
Alexander V. Kildishev* and Vladimir M. Shalaev \\ Birck Nanotechnology Center and School of Electrical and Computer Engineering, Purdue University, \\ West Lafayette, Indiana 47907, USA \\ *Corresponding author: a.v.kildishev@ieee.org
}

Received October 22, 2007; revised November 16, 2007; accepted November 20, 2007; posted November 28, 2007 (Doc. ID 88891); published December 18, 2007

\begin{abstract}
Conceptual studies and numerical simulations are performed for imaging devices that transform a near-field pattern into magnified far-zone images and are based on high-order spatial transformation in cylindrical domains. A lens translating a near-field pattern from an almost circular input boundary onto a magnified far-field image at a flat output boundary is considered. The lens is made of a metamaterial with anisotropic permittivity and permeability both depending on a single "scaling" parameter of the transformation. Open designs of the lens with a truncated body ( $\frac{3}{4}$-body and $\frac{1}{4}$-body lenses) are suggested and analyzed. It is shown that the ideal full lens and the $\frac{3}{4}$-body lens produce identical images. Numerical simulations of $\frac{1}{4}$-body designs indicate that further truncation of the lens could limit its performance. A light concentrator "focusing" far-zone fields into a nanometer-scale area is also considered. (C) 2007 Optical Society of America

OCIS code: 160.4670.
\end{abstract}

Recently, increasing attention has been applied to creating an electromagnetic cloak of invisibility based on various schemes, including anomalous localized resonance [1], dipolar scattering cancellation [2], tunneling light transmittance [3], sensors and active sources [4], and transformation optics [5-8]. The last approach was also employed for demonstrating cloak designs for the visible range $[9,10]$. However, transformation optics can go far beyond just cloaking because it allows one to control the path of light in an unprecedented manner. By creating complex spatial distributions of dielectric permittivity $\epsilon(r)$ and magnetic permeability $\mu(r)$, one can "curve" the optical space in any desired way and mold the flow of light.

The detailed fundamentals of 3D transformation optics have been developed elsewhere [11,12]. A related $2 \mathrm{D}$ design pertinent to an optical hyperlens in concentric circular cylinder domains has also been proposed [13] and validated experimentally $[14,15]$.

In this Letter, we develop the conceptual basis for devices transforming near-field patterns into magnified (beyond the diffraction limit) far-zone images that can be detected with conventional optics. We also propose a "half-space" concentrator for light that is different from the earlier proposed cylindrical concentrator [16] and represents, in a sense, a "reversed" magnifying lens. In contrast with [13-15,17], which dealt with two concentric circular cylinder boundaries, new transformations connect a near-field pattern at an interior, almost circular cylinder, boundary with a far-field pattern at an exterior planar boundary.

In a general cylindrical coordinate system (CCS), $\rho$, $\phi$, and $z$, can be arranged by translating an $x y$-plane map $[x=x(\rho, \phi), y=y(\rho, \phi)]$ perpendicular to itself, and the resulting coordinate system forms families of concentric cylindrical surfaces. Given that $\vec{r}=\hat{\mathbf{x}} x+\hat{\mathbf{y}} y$, the scaling factors of a given CCS are equal to $s_{\rho}$ $=\left|\vec{r}^{(\rho)}\right|, s_{\phi}=\left|\vec{r}^{(\phi)}\right|$, and $s_{z}=1$, where $(.)^{(\xi)}=\partial(.) / \partial \xi$. It is convenient to choose a CCS with $s_{\rho}=s_{\phi}=s$. For the
TM case, the curl of the magnetic field intensity, $\vec{H}$ $=\hat{\mathbf{z}} h$, is $s \nabla h \times \hat{\mathbf{z}}=-h^{(\rho)} \hat{\boldsymbol{\phi}}+h^{(\phi)} \hat{\boldsymbol{\rho}}$ or $-i \omega s d_{\rho} \hat{\boldsymbol{\rho}}-i \omega s d_{\phi} \hat{\boldsymbol{\phi}}$; so the components of the electric displacement vector, $d_{\rho}$ and $d_{\phi}$, are equal to $d_{\rho}=(-i \omega s)^{-1} h^{(\phi)}$ and $d_{\phi}$ $=(i \omega s)^{-1} h^{(\rho)}$, respectively. Transition to $h$ is given by $\left(\epsilon_{\phi}^{-1} d_{\phi}\right)^{(\rho)}-\left(\epsilon_{\rho}^{-1} d_{\rho}\right)^{(\phi)}=i s \omega \epsilon_{0} \mu_{0} \mu h$, and the wave equation is defined by

$$
\left(s^{-1} \epsilon_{\phi}^{-1} h^{(\rho)}\right)^{(\rho)}+\left(s^{-1} \epsilon_{\rho}^{-1} h^{(\phi)}\right)^{(\phi)}+\mu s k_{0}^{2} h=0 .
$$

In a virtual free-space $(\eta, \phi, z)$,

$$
\left(\widetilde{s}^{-1} h^{(\eta)}\right)^{(\eta)}+\left(\widetilde{s}^{-1} h^{(\phi)}\right)^{(\phi)}+\widetilde{s} k_{0}^{2} h=0 .
$$

The simplest approach to transforming concentric cylindrical domains is built on a scaling transformation, $\rho=\rho(\eta)$, where parameter $\eta$ of the initial virtual domain is mapped onto a corresponding parameter $\rho$ in the physical world, keeping the other coordinate of the $x y$-plane $\phi$ intact. Then, Eq. (1) can be rearranged in the following manner using $\rho=\rho(\eta)$ and $f^{(\rho)}=\eta^{(\rho)} f^{(\eta)}$ :

$$
\begin{aligned}
& {\left[\left(\frac{\eta^{(\rho)} \tilde{s}}{\epsilon_{\phi} s}\right) \widetilde{s}^{-1} h^{(\eta)}\right]^{(\eta)}+\left[\left(\frac{\widetilde{s}}{\eta^{(\rho)} s \epsilon_{\rho}}\right) \widetilde{s}^{-1} h^{(\phi)}\right]^{(\phi)}} \\
& +k_{0}^{2}\left(\frac{\mu s}{\eta^{(\rho)} \tilde{s}}\right) \tilde{s} h=0 .
\end{aligned}
$$

To match the above equation for the field inside the lens with that of the free-space in Eq. (2), the terms in parentheses should be equal to unity, i.e., the material of the lens should provide the following local properties:

$$
\epsilon_{\phi}=\eta^{(\rho)} \widetilde{s} / s, \quad \epsilon_{\rho}=\widetilde{s} /\left(s \eta^{(\rho)}\right), \quad \mu=\epsilon_{\phi} .
$$

The aforementioned rules are valid for any device using a concentric mapping of cylindrical domains with either a linear or high-order scaling transform, $\rho(\eta)$, 

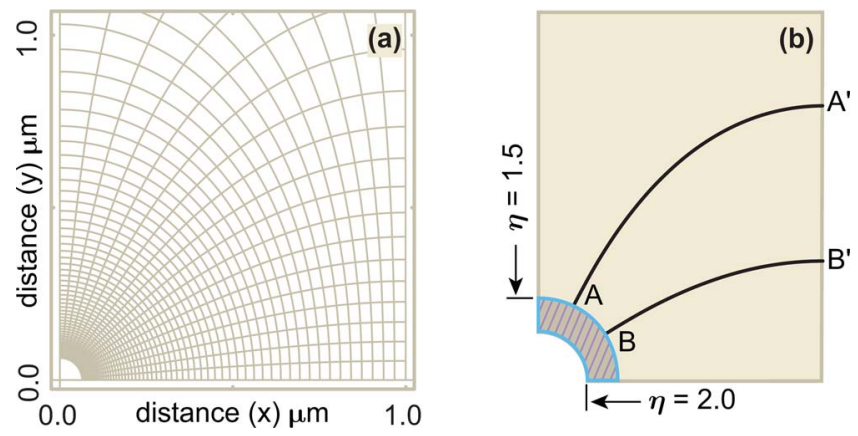

Fig. 1. (Color online) Transformation of concentric cylindrical domains. (a) One-quarter $x y$-map generated by Eqs. (4) with $x_{0}=1 \mu \mathrm{m}, 0 \leqslant \phi \leqslant \pi / 2$, and $0 \leqslant \eta \leqslant 3$. (b) Mapping of virtual domain (hatched quarter-ring) onto the physical domain (solid region), where the shared boundary is at $\rho=\eta$ $=2$. For example, points $\mathrm{A}$ and $\mathrm{B}$ from the curvilinear boundary at $\eta=1.5$ are mapped onto the points $\mathrm{A}^{\prime}$ and $\mathrm{B}^{\prime}$ of the external boundary of the lens at $\rho=0$.

while preserving the common orthogonal parameter $\phi$. For example, consider a CCS generated by the following $x y$-map:

$$
\begin{gathered}
x=\alpha\left[\frac{\pi}{2}-\tan ^{-1}\left(\frac{\sinh \rho}{\cos \phi}\right)\right], \\
y=\frac{\alpha}{2} \ln \left(\frac{\cosh \rho+\sin \phi}{\cosh \rho-\sin \phi}\right),
\end{gathered}
$$

with $s=\alpha \sqrt{2} / \xi_{\rho}$, for $0 \leqslant \rho<\infty$, and $-\pi / 2<\phi<\pi / 2$; here $\alpha=2 x_{0} / \pi$ and $\xi_{\rho}=\sqrt{\cosh 2 \rho+\cos 2 \phi}$.

For the ideal lens, a straightforward linear transform $\rho(\eta)=\tau(\eta-b)$ with $\tau=a(a-b)^{-1}$ gives $\eta=\tau^{-1} \rho+b$, and $\eta^{(\rho)}=\tau^{-1}$. Thus,

$$
\epsilon_{\phi}=\kappa / \tau, \quad \epsilon_{\rho}=\kappa \tau, \quad \mu=\epsilon_{\phi},
$$

where $\kappa=\xi_{\rho} / \xi_{\eta}, \xi_{\eta}=\sqrt{\cosh 2 \eta+\cos 2 \phi}$.

Figure 1(a) depicts a one-quarter $x y$ map generated by Eqs. (4) with $x_{0}=1 \mu \mathrm{m}, 0 \leqslant \phi \leqslant \pi / 2$, and $0 \leqslant \rho \leqslant 3$. Figure 1(b) shows the mapping of a virtual domain (hatched quarter ring) onto a physical domain (solid region) with the shared boundary at $\rho=\eta=2$. Thus, points $A$ and $B$ from the virtual external boundary at $\eta=1.5$ are mapped onto the points $\mathrm{A}^{\prime}$ and $\mathrm{B}^{\prime}$ of the physical external boundary of the lens at $\rho=0$.

Calculation of the material properties requires the inverse transforms, which in this case are given by

$$
\rho=\frac{1}{2} \ln \left(\frac{\cosh \hat{y}+\cos \hat{x}}{\cosh \hat{y}-\cos \hat{x}}\right), \quad \phi=\tan ^{-1}\left(\frac{\sin \hat{x}}{\sinh \hat{y}}\right),
$$

where the scaled coordinates $(\hat{x}, \hat{y})$ are defined as $\hat{x}$ $=x / \alpha$ and $\hat{y}=y / \alpha$.

Finally, the anisotropic permittivity, $\overleftrightarrow{\epsilon}$, is equal to

$$
\overleftrightarrow{\epsilon}=\frac{\kappa}{2}\left[\left(\tau+\tau^{-1}\right) \mathbf{i}+\frac{\tau-\tau^{-1}}{\cosh 2 \hat{y}-\cos 2 \hat{x}} \mathbf{u}\right],
$$

where $\mathbf{i}$ is a $2 \times 2$ idem factor; the components of the
$2 \times 2$ symmetric matrix $\mathbf{u}$ are $u_{x y}=u_{1}$ and $u_{x x}=-u_{y y}$ $=u_{0}, \quad$ with $u_{1}=\sinh 2 \hat{y} \sin 2 \hat{x} \quad$ and $u_{0}=1$ $-\cosh 2 \hat{y} \cos 2 \hat{x}$.

Figure 2 shows a proof-of-concept test of the lens with smoothly changing local properties in accordance with Eqs. (5). The test sources in Fig. 2(a) are two cylindrical segments excited as magnetic line sources with $\lambda=1.55 \mu \mathrm{m}$ at the virtual surface of a circular cylinder with a radius of $152 \mathrm{~nm}$; the angular dimensions of the sources range from $10^{\circ}$ to $40^{\circ}$. In contrast with Fig. 1(a), where the sources give a combined (nonresolved) far-field pattern, in Fig. 2(b) the sources are resolved at the flat edge of the lens.

This closed design of the lens could limit its practical use. To provide better access to the input surface, a part of the lens can be removed. Figure 3 shows the effect of a symmetric truncation of the lens body. Two open designs of the lens are shown in Figs. 3(a) and 3(b), where the shaded sections indicate the lens cross section in both panels. Figure 3(a) depicts the H-field map inside a $\frac{3}{4}$-body lens, while Fig. $3(\mathrm{~b})$ shows the map for $\frac{1}{4}$-body lens. The H-field magnitudes generated by the sources along the flat (output) boundary of the lenses are compared in Fig. 3(c). The $\mathrm{H}$-field at the image plane of the $\frac{3}{4}$-body lens (dashed curve) and the full-lens design (gray solid curve) completely overlap. This good match is almost preserved in the $\frac{1}{2}$-body lens (not shown), but the performance of the lens decreases with further truncation. For example, Fig. 3(c) shows additional artifacts and decreasing intensity at the image boundary.

Finally, a similar approach is taken to make a light-concentrating device, where for the ideal case another common boundary, $\rho=\eta=0$, is used along with the simple transformation $\rho=\tau \eta, \tau=l / b$, which results in mapping a virtual boundary $\eta=b$ onto a physical boundary inside the lens $(\rho=l)$. The transform gives the same formal properties as Eqs. (5).

Figure 4 compares the performance of open designs of light-concentrating devices, shown in Figs. 4(b)-4(d) versus the full-scale concentrator shown in 4(a). All the panels show the time-averaged energy density on a logarithmic scale, while the concentra-
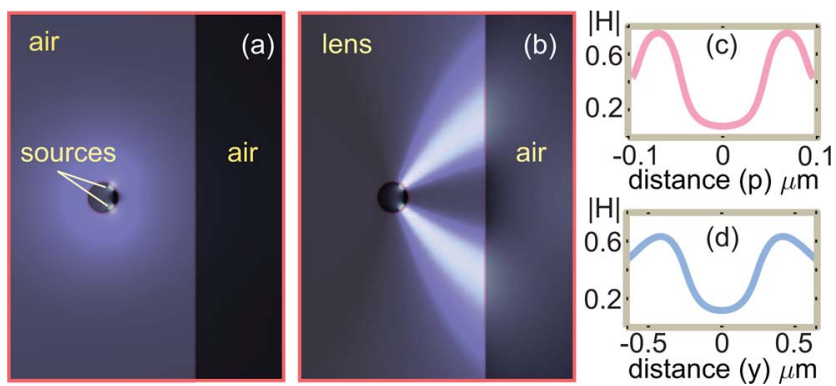

Fig. 2. (Color online) Test of near-to-far-field projection. (a) Magnetic field intensity generated by two coherent test sources in air. (b) Magnetic field intensity generated by the pair of test sources inside and just outside the lens. Shaded areas indicate the lens cross section in (a) and (b). (c) $\mathrm{H}$-field magnitude generated by the sources along the curvilinear (input) surface of the lens, $\rho=\eta=2$. (d) H-field magnitude created by the sources at the flat (output) edge, $\rho=0$. 

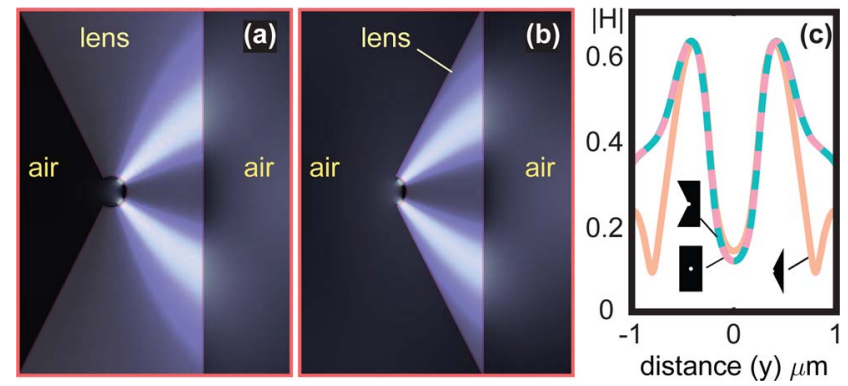

Fig. 3. (Color online) Open designs of the lens. (a) Magnetic field map inside a $\frac{3}{4}$-body lens. (b) Field map inside a $\frac{1}{4}$-body lens. Shaded areas indicate the lens cross-section in (a) and (b). (c) H-field generated by the sources along the flat surface of either the closed or open lens designs.

tors are illuminated by a $750 \mathrm{~nm}$ plane wave propagating from right to left. The geometrical and transform parameters of all the concept devices in Fig. 4 are $x_{0}=1 \mu \mathrm{m}, l=200$, and $b=0.01$. Figures $4(\mathrm{a})$ and 4(b) depict designs with identical performance; the focusing properties of the open design in Fig. 4(b) are not affected by the truncation of the device body. In contrast with the designs of Figs. 4(a) and 4(b), the open designs of Figs. 4(c) and 4(d) demonstrate visible distortion and undesired localization of energy density due to additional interfaces interrupting the smooth transformation of critical spatial modes.

In summary, this Letter deals with proof-of-concept numerical simulations of near-to-far-field magnified imaging and light-concentrating devices built on
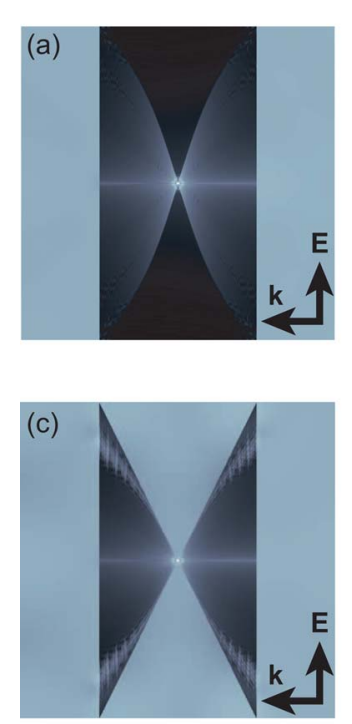
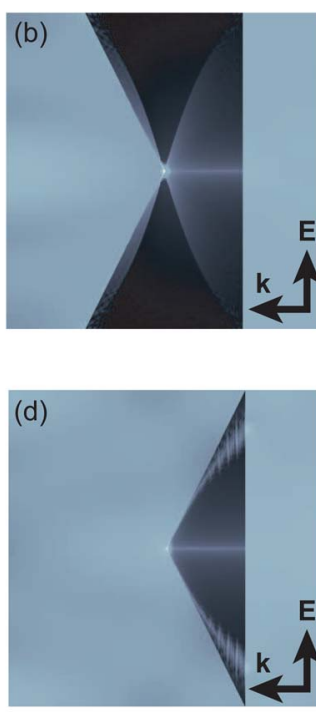

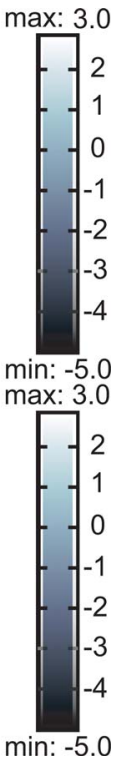

Fig. 4. (Color online) Comparison of the full design of the light-concentrator (a) with an open $\frac{3}{4}$-body design (b). (cc) and (d), $\frac{1}{2}$-body and $\frac{1}{4}$-body designs of the concentrator. All the panels show the time-averaged energy density on a logarithmic scale for a TM-polarized $750 \mathrm{~nm}$ plane wave propagating from right to left. high-order spatial transformations in general cylindrical domains. The primary results are (i) a lens translating a near-field pattern generated at an almost circular input boundary onto a magnified farfield pattern at a flat output boundary and (ii) a light concentrator focusing the energy of a plane-wave input into a subwavelength spot. The lens and the concentrator are modeled out of a metamaterial with anisotropic permittivity and permeability both depending only on a single parameter $(\rho)$ of an orthogonal coordinate system that defines the transformation. Two open designs of the lens with truncated body ( $\frac{3}{4}$-body and $\frac{1}{4}$-body lenses) are studied and compared with a full, closed design. It is shown that the ideal full-lens design and the truncated $\frac{3}{4}$-body lens produce identical images. Similarly, the $\frac{3}{4}$-body light concentrator provides performance comparable with the full body ideal design. Numerical studies of the $\frac{1}{4}$-body lens and concentrator indicate that further truncation of the device could substantially limit its functionality.

The authors are grateful to Narimanov for useful discussions. This work was supported in part by Army Research Office grant W911NF-04-1-0350 and by ARO-MURI award 50342-PH-MUR.

\section{References}

1. G. W. Milton and N. A. P. Nicorovici, Proc. R. Soc. London, Ser. A 462, 3027 (2006).

2. A. Alu and N. Engheta, Phys. Rev. E 72, 016623 (2005).

3. F. J. Garcia de Abajo, G. Gómez-Santos, L. A. Blanco, A. G. Borisov, and S. V. Shabanov, Phys. Rev. Lett. 95, 067403 (2005).

4. D. A. B. Miller, Opt. Express 14, 12457 (2006).

5. J. B. Pendry, D. Schurig, and D. R. Smith, Science 312, 1780 (2006).

6. U. Leonhardt, Science 312, 1777 (2006).

7. A. Greenleaf, M. Lassas, and G. Uhlmann, Bone Marrow Transplant 24, 413 (2003).

8. D. Schurig, J. J. Mock, B. J. Justice, S. A. Cummer, J. B. Pendry, A. F. Starr, and D. R. Smith, Science 314, 977 (2006).

9. W. Cai, U. K. Chettiar, A. V. Kildishev, and V. M. Shalaev, Nat. Photonics 1, 224 (2007).

10. W. Cai, U. K. Chettiar, A. V. Kildishev, and V. M. Shalaev, Appl. Phys. Lett. 91, 111105 (2007).

11. A. J. Ward and J. B. Pendry, J. Mod. Opt. 43, 773 (1996).

12. U. Leonhardt and T. G. Philbin, New J. Phys. 8, 247 (2006).

13. Z. Jacob, L. V. Alekseyev, and E. Narimanov, Opt. Express 14, 8247 (2006).

14. Z. Liu, H. Lee, Y. Xiong, C. Sun, and X. Zhang, Science 315, 1686 (2007).

15. I. I. Smolyaninov, Y.-J. Hung, and C. C. Davis, Science 315, 1699 (2007).

16. M. Rahm, D. Schurig, D. A. Roberts, S. A. Cummer, D. R. Smith, and J. B. Pendry, http://aps. arxiv.org/abs/ 0706.2452 (2007).

17. A. V. Kildishev and E. E. Narimanov, Opt. Lett. 32, 3432 (2007). 